\title{
HEEGAARD SPLITTINGS AND A THEOREM OF LIVESAY
}

\author{
J. H. RUBINSTEIN ${ }^{1}$
}

\begin{abstract}
Let $M$ be a nonorientable 3-manifold which is double covered by $S^{2} \times I$. We give a short proof of the theorem of Livesay [1] that $M$ is homeomorphic to $P^{2} \times I$ (where $P^{2}$ denotes the projective plane).
\end{abstract}

0 . Let $M$ be a compact connected nonorientable 3-manifold with $\partial M$ consisting of two copies of $P^{2}$ and $\pi_{1}(M)=Z_{2}$. Using the analysis of the Heegaard splittings of $S^{3}$ in [4], we prove the following

THEOREM [1]. If the orientable two-fold cover of $M$ is homeomorphic to $S^{2} \times I$ then there is an annulus $A$ embedded in $M$ with $A \cap \partial M=\partial A$ consisting of two simple closed noncontractible curves, one in each component of $\partial M$.

As in [1] the fact that $M$ is homeomorphic to $P^{2} \times I$ follows immediately. We divide the proof of this theorem into a number of steps, working throughout in the PL category.

1. Let $r: M \rightarrow P$ be a retraction such that $r$ restricted to each component of $\partial M$ is a homeomorphism. $r$ can be chosen transverse to a simple closed noncontractible curve $\alpha$ in $P$. Then $r^{-1}(\alpha)$ is a compact 2-manifold embedded in $M$. Exactly as in [1] it follows that $r^{-1}(\alpha)$ contains a component $K$ which is orientable, one-sided and has $\partial K$ equal to two noncontractible simple closed curves, one in each component of $\partial M$.

Let $p: S^{2} \times I \rightarrow M$ be the double covering and let $g: S^{2} \times I \rightarrow S^{2} \times I$ be the covering transformation. Let $L$ denote $p^{-1}(K)$. If $W$ is the closure of a component of $S^{2} \times I-L$, then $W \cup g W=S^{2} \times I$ and $W \cap g W=L$.

Clearly we can assume without loss of generality that $K$ is incompressible, i.e., there is no disk $D$ embedded in $M$ with $D \cap K=\partial D$ and $\partial D$ a noncontractible curve in $K$. Also it will be supposed that genus $K>0$, i.e., $K$ is not an annulus.

2. We show that $K$ incompressible implies $W$ is a handlebody. By [2] there are disjoint simple closed noncontractible curves $C_{1}, \ldots, C_{m}$ in $\partial W$ such that the normal closure of the elements of $\pi_{1}(\partial W)$ given by joining each $C_{i}$ to the base point along some path for $1 \leqslant i \leqslant m$ is $\operatorname{Ker} \Phi$ (where $\Phi: \pi_{1}(\partial W) \rightarrow$ $\pi_{1}(W)$ is induced by the inclusion map).

Received by the editors December 15, 1975.

AMS (MOS) subject classifications (1970). Primary 57A10; Secondary 55A35.

Key words and phrases. Heegaard splitting, system of meridian surfaces, handlebody.

${ }^{1}$ The author held a Melbourne University Research Fellowship during this research.

Copyright $\odot$ 1977, American Mathematical Society 
By [3], $C_{1}, \ldots, C_{m}$ bound disks $D_{1}, \ldots, D_{m}$ in $W$ which can be easily made disjoint from each other, with $D_{i} \cap \partial W=C_{i}$ for all $i$. Without loss of generality we can assume that $C_{1}, \ldots, C_{m}$ lie in $L$. Let $N\left(D_{i}\right)$ (or $N(L)$ ) be a small closed regular neighbourhood of $D_{i}$ (or $L$ ) in $W$ (or $S^{2} \times I$ ) and let $J$ denote the boundary of $g W \cup N(L) \cup N\left(D_{1}\right) \cup \cdots \cup N\left(D_{m}\right)$. Then $J$ is mapped homeomorphically into $M$ by $p$.

Suppose that $J$ is not a union of 2-spheres. Let $X, Y$ be the closures of the components of $M-p(J)$, with $X$ homeomorphic to $W-\operatorname{int} N\left(D_{1}\right)$ $-\cdots-$ int $N\left(D_{m}\right)$ and $Y$ equal to $N(K) \cup p N\left(D_{1}\right) \cup \cdots \cup p N\left(D_{m}\right)$ (where $N(K)=p N(L)$ ). Then there is a simple closed noncontractible curve $C$ in $p(J)$ which contracts in $X$ or $Y$ by [2]. As $C_{1}, \ldots, C_{m}$ give rise to elements of $\pi_{1}(\partial W)$ which normally generate $\operatorname{Ker} \Phi, C$ must contract in $Y$.

Consequently by [3] there is a disk $D$ embedded in $Y$ with $D \cap \partial Y=\partial D=$ $C$. Since $K$ is incompressible, $D \cap K=\varnothing$ can be arranged. But $N(K)-K$ is homeomorphic to $L \times(0,1]$ and so $Y-K$ is homotopically equivalent to $L \cup D_{1} \cup \cdots \cup D_{m}$. This implies that $C$ contracts in $L \cup D_{1} \cup \cdots \cup D_{m}$ and therefore also in $J$, which is a contradiction.

This establishes that $J$ is a union of 2 -spheres and the fact that $W$ is a handlebody follows directly.

3. Let genus $L$ be denoted by $n$. By [4] since $\partial W$ gives a Heegaard splitting of $S^{3}$ with two open 3-cells removed, there is a good system of meridian surfaces $D=D_{1} \cup \cdots \cup D_{n}$ in $W$ and a $D$-coordinate system $\bar{D}=\bar{D}_{1}$ $\cup \cdots \cup \bar{D}_{n}$ in $g W$. As defined in (2.1) of [4], this means that $D, \bar{D}$ are both collections of disjoint disks, such that $C_{i} \cap \bar{C}_{j}$ is a single transverse crossing point for $i=j$ and is empty for $i>j$, where $C_{i}=\partial D_{i}=D_{i} \cap \partial W$ and $\bar{C}_{j}=\partial \bar{D}_{j}=\bar{D}_{j} \cap \partial g W$ for all $i, j$. Without loss of generality we can assume that $C_{i}, \bar{C}_{i}$ are contained in $L$ for all $i$.

Then also $g \bar{D}$ is a good system of meridian surfaces in $W$ and $g D$ is a $g \bar{D}$-coordinate system (with the ordering of the disks reversed). We want to separate the systems $(D, \bar{D})$ and $(g \bar{D}, g D)$ as in Lemma (2.5)(2) of [4]. Clearly we can assume that $D$ and $g \bar{D}$ are transverse and $D \cap g \bar{D}$ consists of arcs only (any simple closed curves can be easily eliminated). Also it can be supposed that $D \cap g \bar{D} \cap g D=\varnothing$.

4. Here $D, \bar{D}$ (or $g \bar{D}, g D$ ) correspond to $v, w$ (or $x, y$ ) in [4]. Let $k$ be an arc of $D_{i} \cap g \bar{D}_{j}$ and let $N(k)$ be a small closed regular neighbourhood of $k$ in $W$.

We can apply the procedure of Lemma $(2.5)(2)$ to the system $D, \bar{D}$ and the arc $k$, producing a new system $D^{\prime}, \bar{D}^{\prime}$. Next the same construction employed on $g \bar{D}^{\prime}, g D^{\prime}$ (or $\bar{D}^{\prime}, D^{\prime}$ with the reverse ordering of disks again) using the arc $k$ (or $g k$ ) gives as outcome a system $g \bar{D}^{\prime \prime}, g D^{\prime \prime}$ (or $\bar{D}^{\prime \prime}, D^{\prime \prime}$ ).

It is easy to see that $D^{\prime \prime}, \bar{D}^{\prime \prime}$ is a good system of meridian surfaces for the splitting given by $W^{\prime \prime}, g W^{\prime \prime}$ and $L^{\prime \prime}=W^{\prime \prime} \cap g W^{\prime \prime}$, where $W^{\prime \prime}=(W-$ int $N(k)) \cup g N(k)$. Also $D^{\prime \prime} \cap g \bar{D}^{\prime \prime}$ has at least one component less than $D \cap g \bar{D}$ and so after a finite number of steps we find that $D \cap g \bar{D}=\varnothing$ is achieved. 
5. We can now utilize the argument in Satz (3.1) of [4]. As in (3.2) of [4], it follows that: By modification of $D$ only we reach that $g D \cap C_{n}$ contains at most one point (and furthermore $D \cap g \bar{D}=\varnothing$ still holds).

The proof is by induction on the number of points in $g D \cap C_{n}$, where $C_{i}$ and $g C_{j}$ are assumed transverse for all $i, j$.

Case 1. Some curve $g C_{j}$ meets $C_{n}$ in at least two points.

By the method in [4], an arc $k$ of $C_{n}$ is found satisfying $\partial k$ is contained in $g C_{j}$ and int $k$ is disjoint from $g D \cup \bar{D}$. Also if $f$ and $h$ are the arcs of $g C_{j}$ with $\partial f=\partial h=\partial k$ then both $g C_{j}^{0}=k \cup f$ and $g C_{j}^{1}=k \cup h$ contract in $g W$. As $D \cap g \bar{D}=\varnothing, k$ is also disjoint from $g \bar{D}$. Therefore $\left(C_{j}^{0} \cup C_{j}^{1}\right) \cap \bar{D}=C_{j} \cap$ $\bar{D}$ and we can suppose that $C_{j}^{0} \cap \bar{C}_{j}=\varnothing$.

We replace $C_{j}$ by $C_{j}{ }^{1}$. Since (int $\left.k\right) \cap \bar{D}=\varnothing, C_{j}^{1}$ bounds a disk $D_{j}^{1}$ in $W$ with $D_{j}^{1} \cap g \bar{D}=\varnothing$. If $j \neq n$ (or $j=n$ ) then $g C_{j}^{1} \cap C_{n}$ (or $g C_{n}^{1} \cap C_{n}^{1}$ ) has at least two points less than $g C_{j} \cap C_{n}$ (or $g C_{n} \cap C_{n}$ ), assuming the intersection is made transverse.

Case 2. $g C_{j}$ meets $C_{n}$ in at most one point for all $j$, but $g D \cap C_{n}$ contains at least two points.

As in [4] there is an arc $k$ of $C_{n}$ with $k \cap \bar{D}=\varnothing$ and $\partial k=k \cap g D=(k$ $\left.\cap g C_{i}\right) \cup\left(k \cap g C_{j}\right)$, for $i<j$ say. Let $C_{j}^{\prime}$ be the curve given by joining $C_{i}$ and $C_{j}$ along $g k$. Then $C_{j}^{\prime} \cap \bar{D}=\left(C_{i} \cup C_{j}\right) \cap \bar{D}$ since $g D \cap \bar{D}=\varnothing$, and $g C_{j}^{\prime} \cap \bar{D}=\varnothing$ because $k \cap \bar{D}=\varnothing$. Finally as $C_{n} \cap g C_{n}$ has an even number of points, $i, j \neq n$. Therefore replacing $C_{j}$ by $C_{j}^{\prime}$, we decrease the number of points in $g D \cap C_{n}$ by one.

6. REMARK. $g D \cap C_{n}=\varnothing$ implies that $C_{n}$ contracts in $g W$ as well as $W$ and so $C_{n}$ is null homologous in $\partial W$, which contradicts $C_{n} \cap \bar{C}_{n}$ is a single transverse crossing point.

So we can assume that $g C_{j} \cap C_{n}$ is a single transverse crossing point and $g C_{i} \cap C_{n}=\varnothing$ for all $i \neq j$, where $j \neq n$. In particular, $g C_{n} \cap C_{n}=\varnothing$. Let $W^{*}=\left(W-\right.$ int $\left.N\left(D_{n}\right)\right) \cup g N\left(D_{n}\right)$ and let $L^{*}=W^{*} \cap g W^{*}$. Clearly $L^{*}$ is a $g$-invariant surface in $S^{2} \times I$.

Let $D^{*}=D-D_{j}-D_{n}$ and let $\bar{D}^{*}=\bar{D}-\bar{D}_{j}-\bar{D}_{n}$. Since $C_{i} \cap g C_{n}=\varnothing$ for all $i \neq j, D^{*}$ is contained in $W^{*}$ with $D^{*} \cap L^{*}=\partial D^{*}$. Also as $\bar{D} \cap g D$ $=\varnothing$ and $\bar{C}_{i} \cap C_{n}=\varnothing$ for all $i<n$, it follows that $\bar{D}^{*}$ is included in $g W^{*}$ and satisfies $\bar{D}^{*} \cap L^{*}=\partial \bar{D}^{*}$. Consequently $D^{*}$ is a good system of meridian surfaces and $\bar{D}^{*}$ is a $D^{*}$-coordinate system for $L^{*}$.

7. Clearly the pair $D^{*}, \bar{D}^{*}$ found in $\S 6$ satisfies $D^{*} \cap g \bar{D}^{*}=\varnothing$. Let us denote $p\left(L^{*}\right)$ by $K^{*}$. Then $K^{*}$ has genus one less than $K$ and using the system $D^{*}, \bar{D}^{*}$ we can repeat the argument in $\S \S 5$ and 6 above. Consequently after a finite number of steps the genus of $K$ is reduced to zero and the theorem is proved.

\section{REFERENCES}

1. G. R. Livesay, Involutions with two fixed points on the three-sphere, Ann. of Math. (2) 78 (1963), 582-593. MR 27 \#5257. 
2. C. D. Papakyriakopoulos, On solid tori, Proc. London Math. Soc. (3) 7 (1957), 281-299. MR 19, 441.

3. , On Dehn's lemma and the asphericity of knots, Ann. of Math. (2) 66 (1957), 1-26. MR 19, 761.

4. F. Waldhausen, Heegaard-Zerlegungen der 3-Sphäre, Topology 7 (1968), 195-203. MR 37 \#3576.

Department of Mathematics, University of Melbourne, Parkville, Victoria, Australia 3052 